\title{
Impact of protease inhibitors on circulating PCSK9 levels in HIV-infected antiretroviral-naive patients from an ongoing prospective cohort
}

\author{
Franck Boccara $^{\mathrm{a}, \mathrm{b}, \mathrm{c}}$, Mathilde Ghislain ${ }^{\mathrm{d}, \mathrm{e}}$, Laurence Meyer ${ }^{\mathrm{d}, \mathrm{e}, \mathrm{f}}$, \\ Cecile Goujard $^{d, e, g}$, Cedric Le May ${ }^{\text {h }}$, Corinne Vigouroux ${ }^{b, c, i}$, \\ Jean P. Bastard ${ }^{\mathrm{c}, j}$, Soraya Fellahi ${ }^{\mathrm{c}, \mathrm{j}}$, Jacqueline Capeau ${ }^{\mathrm{b}, \mathrm{c}}$, \\ Ariel Cohen $^{\mathrm{a}, \mathrm{b}}$, Bertrand Cariou ${ }^{\mathrm{h}, \mathrm{k}}$, the ANRS-COPANA Study Group
}

\begin{abstract}
Objective: The study aims to assess the association between proprotein convertase subtilisin/kexin type 9 (PCSK9), a major regulator of LDL cholesterol (LDL-C) homeostasis, and HIV-related dyslipidaemia in a cohort of HIV-positive (HIV+) patients under protease inhibitors.

Methods: Plasma PCSK9 levels were measured in $103 \mathrm{HIV}+$ patients before and after initiating protease inhibitor-based antiretroviral therapy (ART), and in 90 HIV-negative controls matched for age and sex. PCSK9 was measured by ELISA. HIV+ patients who were not virologically suppressed at follow-up or were on lipid-lowering therapy were excluded.

Results: In HIV+ (median age 36 years; $77.7 \%$ men), PCSK9 levels did not increase after protease inhibitor exposure (median 14 months) $(279.5 \mathrm{ng} / \mathrm{ml}$ before, $289.6 \mathrm{ng} / \mathrm{ml}$ after; $P=0.49$ ) and were significantly elevated versus controls at all timepoints (adjusted $P$ value before and after: $<0.05$ ). After protease inhibitor initiation, total cholesterol, LDL-C and HDL cholesterol levels increased, but LDL-C remained lower versus controls. At baseline, PCSK9 levels were positively associated with immunodeficiency and the severity of HIV disease [HIV-1 viral load $(P=0.01), \mathrm{CD}^{+} \mathrm{T}$-cell count $<200 / \mu \mathrm{l}, P=0.002$ ], stage $C$ HIV disease $(P=0.0002)$. In protease inhibitor-treated patients, PCSK9 levels were no longer associated with HIV-related factors but with total cholesterol $(P=0.0006)$, LDL-C $(P=0.01)$, HDL cholesterol $(P=0.01)$, triglycerides $(P=0.05)$ and glycaemia $(P=0.006)$.
\end{abstract}

Conclusion: PSCK9 levels are elevated in HIV+ patients. In ART-naive patients, the relationship between PCSK9 levels and infection severity suggests an effect of HIV disease. After initiating protease inhibitor-containing ART in virologically suppressed patients, PCSK9 levels were associated with dyslipidaemia similar to controls.

Keywords: dyslipidaemia, HIV, proprotein convertase subtilisin/kexin type 9, protease inhibitor

\footnotetext{
${ }^{a}$ AP-HP, Hôpital Saint-Antoine, Service de cardiologie, 'borbonne-Universités, UPMC université Paris 06, Faculté de médecine, 'INSERM, Saint-Antoine Research Center, UMR S 938, Medical Faculty of Saint Antoine, Paris, ${ }^{d}$ Inserm UMRS1018, CESP, HIV and Pediatry, ${ }^{e}$ Paris-Sud University, ${ }^{\mathrm{f}}$ Department of Public Health, Bicêtre Hospital, APHP, ${ }^{8}$ Department of Internal Medicine, Bicêtre Hospital, AP-HP, Le Kremlin-Bicêtre, ' 'I'institut du thorax, INSERM, CNRS, UNIV Nantes, Nantes, 'AP-HP, Hôpital SaintAntoine, Laboratoire Commun de Biologie et Génétique Moléculaires et Endocrinologie, 'ंAssistance Publique des Hôpitaux de Paris, Hôpital Tenon, UF Bio-marqueurs Inflammatoires et métaboliques, Service de Biochimie, Paris, and 'l'institut du thorax, CHU Nantes, Service de endocrinologie-maladies métaboliques et nutrition, Nantes, France.

Correspondence to Franck Boccara, MD, PhD, Department of Cardiology, Saint Antoine Hospital, University Paris 6, AP-HP, 184 rue du Faubourg St Antoine, 75012 Paris, France.

Tel: +33 14928449; fax: +33 149282683; e-mail: franck.boccara@aphp.fr
} 


\section{Introduction}

HIV-positive $(\mathrm{HIV}+)$ patients are at high risk of coronary heart disease, partly due to atherogenic dyslipidaemia [1]. Mechanisms of HIV-associated dyslipidaemia are complex, and may involve the HIV-infected state and some antiretroviral therapies (ARTs), particularly protease inhibitors. In ART-naive HIV+ patients, LDL cholesterol (LDL-C) levels are often reduced, with an increase in small dense LDL particles [2]. In such patients, the increase in plasma triglycerides may be due to the inflammatory state and notably the increase in TNF $\alpha$ associated with uncontrolled infection and reduced clearance of triglycerides [3]. In contrast, after initiating ART, especially protease inhibitor boosted with ritonavir $(\mathrm{PI} / \mathrm{r})$, total cholesterol (TC), LDL-C and HDL cholesterol (HDL-C) levels increase, and also often triglyceride levels [4]. These increased levels may return to the baseline state after immunovirological control of HIV infection. However, HDL-C usually remains low, with moderate-to-high levels of triglycerides. Certain ARTs, particularly first-generation $\mathrm{PI} / \mathrm{r}$, have been clearly shown to be involved in this abnormal state [1]. Before ART initiation, low levels of TC, LDL-C and HDL-C are related to HIV severity. However, high plasma lipid levels are related to both the efficacy of antiretrovirals in HIV suppression and the effect of certain ART.

Proprotein convertase subtilisin/kexin type 9 (PCSK9) acts as a natural inhibitor of the LDL-receptor pathway. A0fter intracellular autocleavage, PCSK9 is secreted by the liver, binds to the epidermal growth factor repeat A domain of the LDL receptor and induces its degradation by forcing its shuttling to lysosomes. Although PCSK9 gain-of-function mutations cause autosomal-dominant hypercholesterolaemia and premature atherosclerosis, loss-of-function mutations lead to low levels of plasma LDL-C and confer high protection against coronary heart disease [5-8]. Pharmacological inhibition with PCSK9 monoclonal antibodies (e.g. alirocumab and evolocumab) is effective in lowering LDL-C in patients with familial hypercholesterolaemia or atherosclerosis who fail to achieve optimal LDL-C targets despite maximally tolerated doses of statins [9]. Recently, the large cardiovascular outcomes trial FOURIER demonstrated a significant reduction in the rate of major cardiovascular events with evolocumab versus placebo [10].

As PCSK9 expression is induced in some inflammatory states, such as lipopolysaccharide injection in mice or septic shock in humans, it could be envisaged that PCSK9 plays a role in HIV-associated dyslipidaemia $[11,12]$. To date, only one study has focused on circulating PCSK9 levels in HIV+ patients and HIV/hepatitis $\mathrm{C}$ virus $(\mathrm{HCV})$ coinfected patients, showing what the authors called a 'PCSK9-lipid paradox' [13]. Indeed, in their observational, cross-sectional study, the authors found that $\mathrm{HIV} / \mathrm{HCV}$ coinfected patients had increased plasma PCSK9 levels, despite reduced LDL-C levels, compared with HIV-monoinfected patients and HIVnegative (HIV-) controls. However, 21\% of coinfected patients were taking statins [13], which increase circulating PCSK9 levels [14], and 22.5\% were not on ART. In immunovirologically controlled HIV+ patients, PCSK9 levels did not differ from controls [13]. Thus, it is important to further evaluate how PCSK9 plasma level is regulated in HIV + patients before and after ART initiation.

To that end, we conducted the Agence de Recherche sur le SIDA et les Hépatites virales (ANRS)-COPANA (Agence de Recherche sur le SIDA et les Hépatites virales-Cohort of HIV-infected Patients with No Antiretroviral Treatment At Baseline)-PCSK9 substudy to assess the impact of first-line ART including a $\mathrm{PI} / \mathrm{r}$, on plasma PCSK9 levels and to identify factors associated with circulating PCSK9 level in ART-naive and treated $\mathrm{HIV}+$ patients.

\section{Methods}

\section{Study population}

ANRS-COPANA is an ongoing prospective cohort study being conducted in 37 hospitals in France. Eighthundred recently diagnosed ( $<12$ months) ART-naive HIV+ adults were recruited between 2004 and 2008 with semiannual follow-up. The cohort is funded by Institut National de la Santé et de la Recherche Médicale-ANRS and the study was approved by the Paris-Cochin Ethics Committee in July 2003 [15]. The research was conducted in accordance with the Declaration of Helsinki. All participants gave their written informed consent.

Sociodemographic, clinical and biological data were collected at enrolment and every 6 months thereafter. Each patient's history of AIDS-defining illnesses, cardiovascular disease, cancer, diabetes mellitus or other diseases, hepatitis B and HCV serostatus were recorded, along with $\mathrm{CD}^{+}$and $\mathrm{CD}{ }^{+}{ }^{-}$-cell counts and plasma HIV-1 RNA viral load. Fasting TC, HDL-C, LDL-C, triglycerides and fasting plasma glucose levels were measured using standard procedures in each centre at least once a year. Frozen plasma and serum were stored at each yearly visit.

This substudy involves ART-naive HIV+ adults who initiated ART including a PI/r and maintained their initial therapy for at least 1 year. Eligibility criteria for inclusion in the present substudy required each patient to have frozen serum and plasma samples at ART initiation and follow-up, and evaluation of lipid variables. HIV+ patients were excluded if they were on lipid-lowering therapy (i.e. statins, fibrates, niacin or ezetimibe), had HIV viral load more than 400 copies $/ \mathrm{ml}$ or had three HIV viral loads more than 50 copies $/ \mathrm{ml}$ 
during study follow-up (Fig. 1). A control group of HIV - patients, matched for age ( \pm 5 years) and sex, was composed from blood donors from the Centre de Transfusion Sanguine in Nantes, France.

\section{Biomarker measurement}

Laboratory data were collected before ART initiation and during treatment. Cryopreserved serum and plasma were used for centralized measurements at Tenon Hospital Biochemistry Department (Paris, France). Plasma glucose (hexokinase) and insulin (chemiluminescence immunoassay) were measured on the Architect Ci8200 analyzer (Abbott Diagnostics, Chicago, Illinois, USA). High-sensitivity C-reactive protein (hs-CRP) was measured by immunonephelometry on an IMMAGE analyzer (Beckman-Coulter, Brea, California, USA). High-sensitivity IL-6 (hs-IL-6) and leptin were measured with ELISAs (Quantikine; R\&D Systems, Oxford, UK). The Homeostasis Model Assessment of Insulin Resistance (HOMA-IR) was defined as HOMA-IR $=($ glucose $\times$ insulin $) / 22.5$ [16]
Adiponectin was measured by ELISA (ALPCO, Salem, New Hampshire, USA). Diabetes was recorded if the patient was receiving antidiabetic treatment or if the fasting plasma glucose level was at least $7 \mathrm{mmol} / \mathrm{l}$. Hypertension was defined if the patient received antihypertensive drugs or had SBP at least $140 \mathrm{mmHg}$ and/or DBP at least $90 \mathrm{mmHg}$. Obesity was defined as BMI more than $30 \mathrm{~kg} / \mathrm{m}^{2}$. ART regimens and treatments for comorbidities were recorded every 6 months.

Fasting plasma PCSK9 levels were assayed in duplicate using a commercially available quantitative sandwich ELISA assay (Circulex CY-8079; CycLex Co, Nagano, Japan) and following the manufacturer's instructions, as described [14].

\section{Endpoints}

The primary objective was to evaluate the impact of the initiation of a first $\mathrm{PI} / \mathrm{r}$-containing ART regimen on PCSK9 levels in ART-naive HIV+ patients. Our primary outcome measure was the mean per cent change

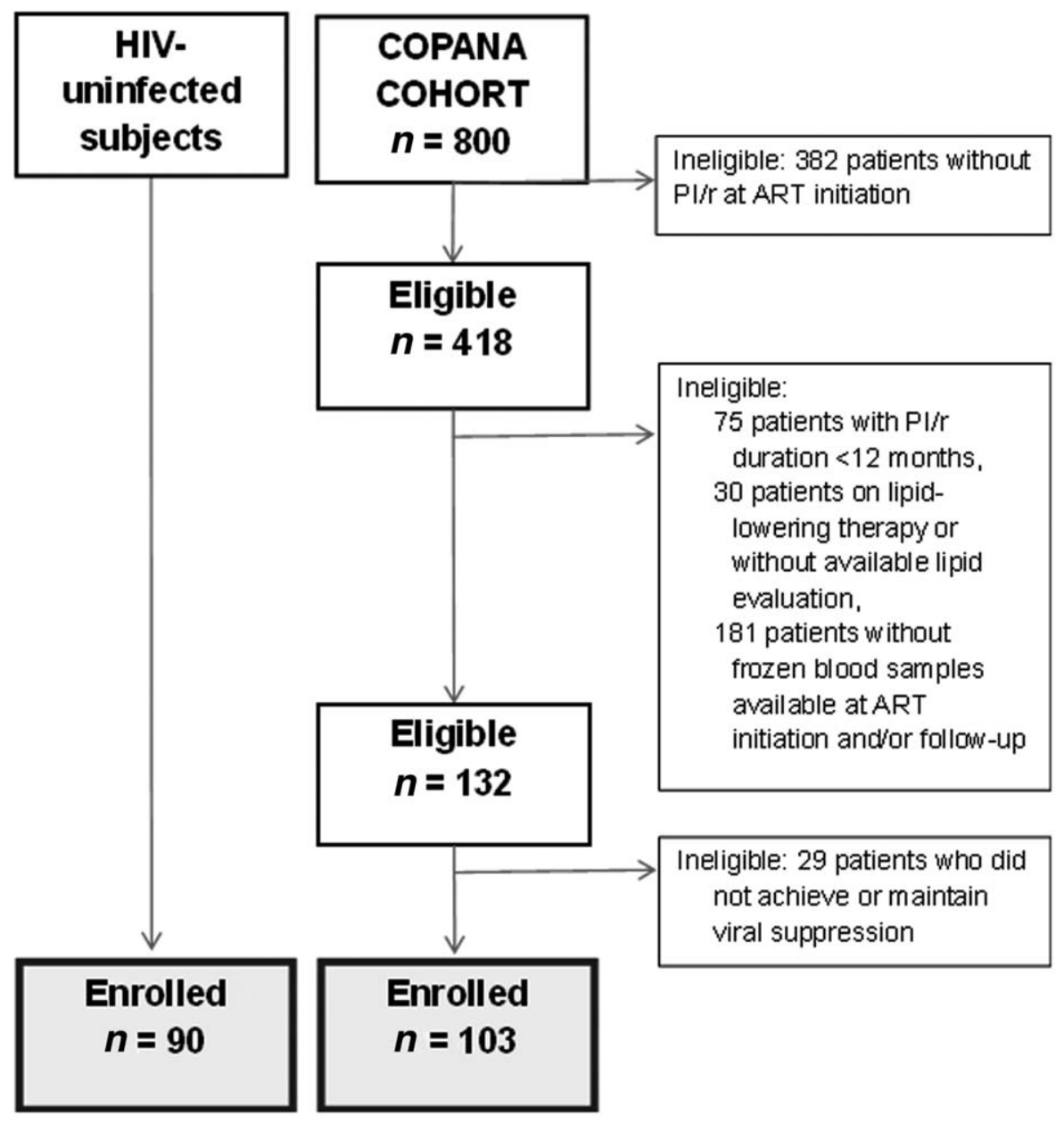

Fig. 1. Consort diagram. ART, antiretroviral therapy; COPANA, Cohort of HIV-infected Patients with No Antiretroviral Treatment At Baseline; $\mathrm{Pl} / \mathrm{r}$, protease inhibitors boosted with ritonavir. 
in PCSK9 plasma levels after initiation of ART including a $\mathrm{PI} / \mathrm{r}$ : values at ART initiation and 1 year later were compared. The secondary objectives were to determine the association between plasma PCSK9 levels and biological variables, in particular lipids, glucose/insulin and inflammatory variables; to compare the change in inflammatory markers/adipokines (hs-IL-6, hs-CRP, leptin, adiponectin) and circulating PCSK9 between baseline (ART-naive HIV+ patients) and 1 year after ART initiation; and to compare plasma PCSK9 levels between HIV - controls and HIV+ patients before and after ART initiation. The secondary outcome measures were the correlations between plasma PCSK9 levels under PI/r and lipid variables (TC, LDL-C, HDL-C, triglycerides) and other variables (fasting plasma glucose and insulin, HOMA-IR); the association between inflammatory markers and PCSK9 levels at baseline and on PI/r therapy; and the comparisons of PCSK9 levels between HIV - patients and HIV + patients before and after ART initiation.

\section{Statistical analyses}

Continuous variables are reported as median [interquartile range (IQR)] and categorical variables as frequency (percentage). $P$ values were obtained from paired $t$ tests and $\mathrm{McNemar}$ or McNemar-Bowker chi-square tests with $P$ less than 0.05 indicating statistical significance. Spearman's correlation coefficients were used to test the association between PCSK9 levels and metabolic variables at $\mathrm{PI} / \mathrm{r}$ initiation and at least 12 months afterwards $(r)$. Multivariable linear regression models were used to examine the influence of age and different metabolic variables on PCSK9 levels before and at least 12 months after initiating ART containing $\mathrm{PI} / \mathrm{r}$.

\section{Results}

\section{Characteristics of the cohort before and after a median exposure to antiretroviral therapy of 14 months}

Of the 800 patients in the COPANA cohort, $103 \mathrm{HIV}+$ patients were eligible for enrolment (Fig. 1). The characteristics of the HIV+ patients before and after ART initiation are detailed in Table 1. In brief, patients were young (median age $=36$ years, $\mathrm{IQR}=30-45$ ), $75.7 \%$ were men and $61.2 \%$ were white. No patients had known cardiovascular disease, $30.1 \%$ were current smokers, $17.5 \%$ had hypertension and 3.9\% had diabetes mellitus at ART initiation.

The most frequently prescribed $\mathrm{PI} / \mathrm{r}$-containing ART was lopinavir/ritonavir (45.6\%), followed by atazanavir/ritonavir (30.1\%), darunavir/ritonavir (11.7\%), fosamprenavir/ritonavir (10.7\%) and saquinavir/ritonavir (1.9\%). After a median exposure to $\mathrm{PI} / \mathrm{r}$-containing ART of 14 months (IQR $=11.5-20.0)$, median $\mathrm{CD}^{+}$cell count increased from 288 to $481 / \mu l \quad(P<0.0001)$ and $99.0 \%$ of patients had an undetectable viral load $(<50$ copies $/ \mathrm{ml})$.

\section{Plasma lipid and proprotein convertase subtilisin/kexin type 9 levels in HIV-positive versus HIV-negative patients}

Compared with ART-naive HIV+ patients, HIVcontrols had significantly higher levels of TC, HDL-C and LDL-C (all $P<0.0001)$, and a trend for lower plasma PCSK9 levels $(P=0.07)$ (Table 2). Plasma PCSK9 levels were significantly higher in ART-naive HIV+ patients than HIV - controls after adjustment for age $(P=0.04)$ and LDL-C $(P=0.02)$. ART treated HIV+ patients exhibited higher levels of plasma PCSK9 and triglycerides, and lower HDL-C levels as compared with HIVcontrols. These differences were maintained after adjustment for lipid variables.

Furthermore, we observed significant differences in plasma PCSK9 levels between protease inhibitor drugs at ART initiation but not during exposure to ART (data not shown).

\section{Change in metabolic variables, adipokines, inflammatory biomarkers and proprotein convertase subtilisin/kexin type 9 before and after a median exposure to antiretroviral therapy of 14 months}

Most lipid variables increased significantly during $\mathrm{PI} / \mathrm{r}$ exposure: TC from 4.2 to $5.1 \mathrm{mmol} / 1 \quad(P<0.0001)$, LDL-C from 2.6 to $3.1 \mathrm{mmol} / 1(P<0.001)$ and HDL-C from 1.1 to $1.2 \mathrm{mmol} / \mathrm{l}(P=0.0001)$, whereas the increase in plasma triglycerides did not reach statistical significance (Table 2). Plasma PCSK9 levels remained stable ( 279.5 versus $289.6 \mathrm{ng} / \mathrm{ml}, P=0.49$ ), thus following a different course than LDL-C (Table 2 and Fig. 2). In addition, changes in plasma PCSK9 levels during exposure to ART were not statistically significant irrespective of the PI/r drug (Supplementary Table S1, http://links.lww.com/QAD/B153).

In treated HIV+ patients, BMI $(P=0.002)$ and fasting plasma glucose levels $(P=0.05)$ increased modestly (Table 1 and Table 2, respectively), whereas no change was observed for insulin $(7.2$ versus $8.1 \mathrm{mUI} / \mathrm{ml}$, $P=0.88)$ or HOMA-IR (1.48 versus $1.73, P=0.46)$, a surrogate of insulin resistance assessment.

Plasma levels of leptin $(P=0.007)$ and the leptin/ adiponectin ratio $(P=0.008)$, increased after initiating ART, whereas adiponectin tended to increase $(P=0.07)$. After ART initiation, plasma levels of leptin significantly increased and levels of adiponectin tended to increase $(P=0.007$ and 0.07 , respectively). The leptin/adiponectin ratio also increased indicating a proportionally larger rise of leptin levels $(P=0.008)$. No change was observed for circulating hs-CRP and hs-IL-6 (Table 2). 
Table 1. Demographic and clinical characteristics of HIV-positive patients before and after initiating protease inhibitors boosted with ritonavircontaining antiretroviral therapy.

\begin{tabular}{|c|c|c|c|}
\hline Variable & Before ART, $n=103$ & After ART, $n=103$ & $P$ value \\
\hline Age (years) & $36(30-45)$ & & \\
\hline Women, $n(\%)$ & $25(24.3)$ & & \\
\hline \multicolumn{4}{|l|}{ Country of birth, $n(\%)$} \\
\hline White & $63(61.2)$ & & \\
\hline Sub-Saharan Africa & $32(31.1)$ & & \\
\hline Other & $8(7.7)$ & & \\
\hline BMI $\left(\mathrm{kg} / \mathrm{m}^{2}\right)(96 / 92)$ & $23.0(20.6-25.6)$ & $23.3(21.4-26.1)$ & 0.002 \\
\hline Waist: hip circumference $(91 / 89)$ & $0.88(0.85-0.94)$ & $0.91(0.86-0.95)$ & 0.004 \\
\hline Current smoker, $n(\%)$ & $31(30.1)$ & $31(30.1)$ & 1.0 \\
\hline Hypertension $^{\mathrm{a}}, n(\%)$ & $18(17.5)$ & $16(15.5)$ & 0.56 \\
\hline Diabetes $^{\mathrm{b}}, n(\%)$ & $4(3.9)$ & $4(3.9)$ & 1.0 \\
\hline Time since HIV diagnosis (months) & $8.5(2.5-23.9)$ & & \\
\hline Duration of $\mathrm{Pl} / \mathrm{r}$ exposure (months) & - & $14(11.5-20.0)$ & \\
\hline Stage C disease, $n(\%)$ & $6(5.8)$ & $7(6.8)$ & 0.32 \\
\hline Hepatitis C positive serology, $n(\%)$ & $5(4.9)$ & $5(4.9)$ & 1.0 \\
\hline HbS antigen $\mathrm{B}, n(\%)$ & $1(1.0)$ & $1(1.0)$ & 1.0 \\
\hline $\mathrm{CD}^{+}{ }^{+}$-cell count $($cells/ $\mu \mathrm{l})(103 / 102)$ & $288(220-387)$ & $481(372-557)$ & $<0.0001$ \\
\hline $\mathrm{CD}^{+} \mathrm{T}$-cell count $($ cells/ $\mu \mathrm{l})(102 / 99)$ & $777(568-1142)$ & $707(516-823)$ & 0.07 \\
\hline $\mathrm{CD}^{+} / \mathrm{CD}^{+}{ }^{+}$ratio $(102 / 99)$ & $0.39(0.26-0.47)$ & $0.73(0.50-0.97)$ & $<0.0001$ \\
\hline $\mathrm{CD}^{+}{ }^{+}$T-cell count, $n(\%)(103 / 102)$ & & & $<0.0001$ \\
\hline$<200$ & $22(21.4)$ & $5(4.9)$ & \\
\hline$\geq 200$ to $<350$ & $47(45.6)$ & $17(16.5)$ & \\
\hline$\geq 350$ to $<500$ & $24(23.3)$ & $37(35.9)$ & \\
\hline$\geq 500$ & $10(9.7)$ & $44(42.7)$ & \\
\hline Undetectable viral load (<50 copies/ml), $n(\%)$ & $0(0.0)$ & $102(99.0)$ & - \\
\hline Viral load $(\mathrm{log} / \mathrm{ml})$ & $4.8(4.1-5.2)$ & $1.3(1.0-1.3)$ & $<0.0001$ \\
\hline
\end{tabular}

Data are medians and $25-75$ th percentiles or $n(\%)$. Numbers in brackets $(n / n)$ indicate number of patients with available data in each group. $P$ values are obtained from paired $t$ test and the McNemar or McNemar-Bowker chi-square test. ART, antiretroviral therapy; HbS, hepatitis B surface antigen; $\mathrm{Pl} / \mathrm{r}$, protease inhibitors boosted with ritonavir.

${ }^{a}$ Treated for hypertension or SBP more than $140 \mathrm{mmHg}$ or DBP more than $90 \mathrm{mmHg}$.

${ }^{\mathrm{b}}$ Treated for diabetes or two consecutive fasting blood glucose at least $7 \mathrm{mmol} / \mathrm{l}$.

${ }^{c}$ When the viral load was undetectable, half of the assay technique detection threshold was applied.

Table 2. Comparison of proprotein convertase subtilisin/kexin type 9, metabolic variables, adipokines and inflammatory marker levels between the antiretroviral therapy-naive HIV-positive, HIV-positive under protease inhibitors boosted with ritonavir and HIV-negative groups.

\begin{tabular}{|c|c|c|c|c|c|c|}
\hline & $\begin{array}{c}\text { ART-naive HIV +, } \\
n=103\end{array}$ & $\begin{array}{l}\mathrm{HIV}+\text { on } \mathrm{Pl} / \mathrm{r} \\
\quad n=103\end{array}$ & $\begin{array}{c}\text { HIV- controls, } \\
\quad n=90\end{array}$ & $P^{*}$ & $P^{* *}$ & $P^{* * *}$ \\
\hline Women, $n(\%)$ & $25(24.3)$ & $25(24.3)$ & $25(27.8)$ & - & 0.58 & 0.58 \\
\hline Age (years) & $36(30-45)$ & $38(32-48)$ & $42(35-47)$ & - & 0.05 & 0.60 \\
\hline PCSK9 levels (ng/ml) (100/100/90) & $279.5(218.2-372.8)$ & $289.6(217.8 ; 381.0)$ & $269.5(223.0-341.8)$ & 0.49 & $0.07^{* * * *}$ & 0.02 \\
\hline Fasting plasma glucose $(\mathrm{mmol} / \mathrm{l})(103 / 100 / 90)$ & $4.8(4.4-5.2)$ & $4.9(4.5-5.3)$ & $4.9(4.5-5.2)$ & 0.05 & 0.65 & 0.23 \\
\hline Total cholesterol (mmol/l) (102/101/90) & $4.2(3.6-4.8)$ & $5.1(4.3-5.8)$ & $5.3(4.8-6.0)$ & $<0.0001$ & $<0.0001$ & 0.11 \\
\hline HDL-cholesterol (mmol/l) (101/99/90) & $1.1(0.9-1.3)$ & $1.2(1.1-1.5)$ & $1.5(1.2-1.7)$ & $<0.0001$ & $<0.0001$ & 0.0003 \\
\hline LDL-cholesterol (mmol/l) (101/99/ 89) & $2.6(2.0-3.0)$ & $3.1(2.5-3.9)$ & $3.4(2.7-3.9)$ & 0.001 & $<0.0001$ & 0.09 \\
\hline Triglycerides (mmol/l) (96/103/90) & $1.1(0.8-1.6)$ & $1.3(1.0-1.9)$ & $1.2(0.8-1.3)$ & 0.14 & 0.10 & 0.03 \\
\hline Leptin (ng/ml) (102/101) & $3.0(1.2-6.7)$ & $4.1(1.7-7.6)$ & NA & 0.007 & & \\
\hline Adiponectin (mg/l) (102/101) & $5.0(3.6-7.1)$ & $5.3(3.6-7.3)$ & NA & 0.07 & & \\
\hline Leptin/adiponectin ratio $\times 10^{3}(102 / 101)$ & $0.6(0.3-1.6)$ & $0.8(0.3-1.6)$ & NA & 0.008 & & \\
\hline hs-CRP (mg/l) (101/96) & $2.3(1.2-4.2)$ & $1.6(0.8-3.3)$ & NA & 0.78 & & \\
\hline hs-IL-6 (pg/ml) (102/101) & $1.6(1.1-2.5)$ & $1.4(1.0-2.2)$ & NA & 0.16 & & \\
\hline
\end{tabular}

Data are medians and $25-75$ th percentiles. Numbers in brackets $(n / n / n)$ indicate number of patients with available data in each group. ART, antiretroviral therapy; HDL-C, HDL cholesterol; HIV+, HIV-positive; hs-CRP, high-sensitivity C-reactive protein; hs-IL-6, high-sensitivity IL-6; LDL-C, LDL cholesterol; NA, not available; PCSK9, proprotein convertase subtilisin/kexin type 9; PI/r, protease inhibitors boosted with ritonavir.

${ }^{*}$ Comparison between naive HIV+ versus HIV+ under Pl/r: $P$ values are obtained from paired $t$ test.

${ }^{*}$ Comparison between naive HIV+ and controls: $P$ values are obtained from $t$ test.

${ }^{* * *}$ Comparison between HIV + under $\mathrm{Pl} / \mathrm{r}$ and controls: $P$ values are obtained from $t$ test.

${ }^{* * * *} P$ value $=0.04$ when adjusted for age $(P=0.04)$ or $\operatorname{LDL}-\mathrm{C}(P=0.02)$. 


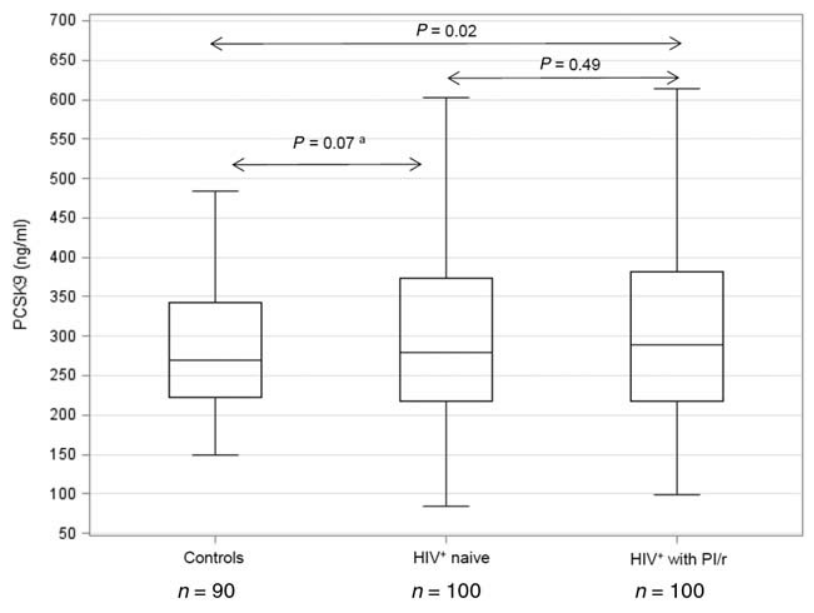

Fig. 2. Comparison of proprotein convertase subtilisin/kexin type 9 levels before and after protease inhibitors boosted with ritonavir exposure in the HIV-positive group and with the HIV-negative control group. ART, antiretroviral therapy; LDL-C, LDL cholesterol; PCSK9, proprotein convertase subtilisin/kexin type 9; $\mathrm{Pl} / \mathrm{r}$, protease inhibitors boosted with ritonavir. All $P$ values are obtained from the $t$ test. $* P<0.05$ when adjusted for age $(P=0.04)$ or LDL-C $(P=0.02)$.

\section{Association of plasma proprotein convertase subtilisin/kexin type 9 with immunologic and metabolic variables before and after a median exposure to antiretroviral therapy of 14 months} Before ART initiation, plasma PCSK9 levels were positively correlated with HIV-1 viral load $(r=+0.24$, $P=0.01)$ and triglycerides $(r=+0.29, \quad P=0.005)$ (Table 3).

No statistically significant correlations were observed between plasma PCSK9 levels and evolution of inflammatory markers, adipokines levels and metabolic variables (Supplementary Table S2, http://links.lww.com/QAD/B153). In addition, we did not observe a correlation between change in PCSK9 and change in $\mathrm{CD} 4^{+}$T-cell count $(r=-0.02, P$ value $=0.81)$.

In univariate linear regression, PCSK9 level before ART initiation was associated with $\mathrm{CD} 4{ }^{+} \mathrm{T}$-cell count less than $200 / \mu 1(P=0.002)$, HIV stage $C$ disease $(P=0.0002)$, and plasma triglycerides more than $1.7 \mathrm{mmol} / 1$ $(P<0.0001)$ (Table 4). These associations remained significant in the multivariate model (Supplementary Table S3, http://links.lww.com/QAD/B153).

Predictors of plasma PCSK9 in virologically suppressed HIV+ patients were markedly different after ART initiation. After ART initiation, PCSK9 levels were positively and significantly correlated with concomitant metabolic variables including plasma lipids: TC $(r=+0.34, P=0.0006)$, LDL-C $(r=+0.27, P=0.01)$ and to a lesser extent HDL-C $(r=+0.17, P=0.01)$ and
Table 3. Spearman correlations of proprotein convertase subtilisin/ kexin type 9 before and after antiretroviral therapy initiation with immunological, metabolic and inflammatory variables (quantitative data).

\begin{tabular}{|c|c|c|c|c|}
\hline \multirow[b]{2}{*}{ Variables } & \multicolumn{2}{|c|}{$\begin{array}{c}\text { Before ART } \\
\text { initiation }\end{array}$} & \multicolumn{2}{|c|}{$\begin{array}{l}\text { After ART } \\
\text { initiation }\end{array}$} \\
\hline & $r$ & $P$ value & $r$ & $P$ value \\
\hline Age at ART initiation & +0.10 & 0.32 & +0.10 & 0.34 \\
\hline HiV-1 viral load ${ }^{\mathrm{a}}$ (copies/ml) & +0.24 & 0.01 & - & - \\
\hline $\mathrm{CD}^{+}{ }^{+}$-cell count $(\mathrm{cell} / \mathrm{s} / \mu \mathrm{l})$ & -0.16 & 0.11 & -0.11 & 0.29 \\
\hline $\mathrm{CD}^{+}{ }^{+}$-cell count $($cells/ $\mu \mathrm{l})$ & -0.005 & 0.96 & +0.06 & 0.56 \\
\hline $\mathrm{CD}^{+} / \mathrm{CD}^{+}{ }^{+} \mathrm{T}$-cell count & -0.18 & 0.08 & -0.10 & 0.34 \\
\hline Weight (kg) & -0.03 & 0.79 & +0.09 & 0.42 \\
\hline $\operatorname{BMI}\left(\mathrm{kg} / \mathrm{m}^{2}\right)$ & -0.11 & 0.29 & +0.10 & 0.36 \\
\hline Waist/hip circumference ratio & -0.05 & 0.62 & +0.07 & 0.51 \\
\hline Triglycerides (mmol/l) & +0.29 & 0.005 & +0.20 & 0.05 \\
\hline LDL-C (mmol/l) & +0.06 & 0.57 & +0.27 & 0.01 \\
\hline $\mathrm{HDL}-\mathrm{C}(\mathrm{mmol} / \mathrm{l})$ & -0.02 & 0.82 & +0.17 & 0.01 \\
\hline Total cholesterol $(\mathrm{mmol} / \mathrm{l})$ & +0.13 & 0.20 & +0.34 & 0.0006 \\
\hline Fasting plasma glucose $(\mathrm{mmol} / \mathrm{l})$ & +0.15 & 0.13 & +0.27 & 0.006 \\
\hline Insulin (mUl/ml) & +0.07 & 0.49 & +0.21 & 0.04 \\
\hline HOMA-IR & +0.09 & 0.36 & +0.23 & 0.02 \\
\hline hs-CRP (mg/l) & -0.04 & 0.69 & -0.08 & 0.42 \\
\hline hs-IL-6 (pg/ml) & +0.13 & 0.21 & +0.08 & 0.45 \\
\hline Leptin $(\mathrm{ng} / \mathrm{ml})$ & -0.009 & 0.93 & +0.16 & 0.12 \\
\hline Adiponectin (mg/l) & +0.04 & 0.69 & -0.02 & 0.87 \\
\hline
\end{tabular}

ART, antiretroviral therapy; HDL-C, HDL cholesterol; HOMA-IR, Homeostasis Model Assessment of Insulin Resistance; hs-CRP, high-sensitivity C-reactive protein; hs-IL-6, high-sensitivity IL-6; LDL-C, LDL cholesterol.

ahen the viral load was undetectable, half of the assay technique detection threshold was applied.

triglycerides $(r=+0.20, \quad P=0.05)$, and also fasting plasma glucose $(r=+0.27, P=0.006)$ and HOMA-IR $(r=+0.23, P=0.02)$ (Table 3). Among HIV+ patients under protease inhibitors, circulating PCSK9 levels were associated with TC more than $5.2 \mathrm{mmol} / 1(P=0.007)$, triglycerides more than $1.7 \mathrm{mmol} / 1 \quad(P=0.009)$ and fasting plasma glucose more than $5.6 \mathrm{mmol} / 1(P=0.03)$ in univariate linear regression (Table 4).

After adjusting for fasting plasma glucose level more than $5.6 \mathrm{mmol} / 1$, the associations of circulating PCSK9 levels with lipids remained statistically significant (Supplementary Table S3, http://links.lww.com/QAD/B153).

No statistically significant correlations were observed between plasma PCSK9 levels and inflammatory markers (hs-CRP and hs-IL-6) or adipokine levels (leptin and adiponectin) in HIV + patients either before or after ART initiation (Table 3).

Furthermore, we explored the associations of plasma PCSK9 levels in patients under ART with different variables, before and after ART, and we adjusted on PCSK9 level before ART. In patients taking protease inhibitors, we observed PCSK9 levels were associated with HOMA-IR more than 2.5 at ART initiation $(P=0.03)$ and were associated with cholesterol more than 
Table 4. Univariate linear regression of proprotein convertase subtilisin/kexin type 9 before and after antiretroviral therapy with immunologic, metabolic and inflammatory variables before and under antiretroviral therapy.

\begin{tabular}{|c|c|c|c|c|}
\hline \multirow[b]{2}{*}{ Variables } & \multicolumn{2}{|c|}{ PCSK9 before ART } & \multicolumn{2}{|c|}{ PCSK9 under $\mathrm{Pl} / \mathrm{r}$} \\
\hline & $\beta$ & $P$ value & $\beta$ & $P$ value \\
\hline Women & +2.22 & 0.95 & & \\
\hline Born in sub-Saharan Africa & -44.9 & 0.14 & & \\
\hline $\mathrm{CD}^{+}{ }^{\mathrm{T}}$-cell count $<200 / \mu \mathrm{l}$ & +102.0 & 0.002 & +15.8 & 0.83 \\
\hline Obesity $^{\mathrm{a}}$ & -34.7 & 0.53 & -3.1 & 0.96 \\
\hline Total cholesterol $>5.2 \mathrm{mmol} / \mathrm{l}$ & +34.4 & 0.43 & +86.7 & 0.007 \\
\hline LDL-C > $4.1 \mathrm{mmol} / \mathrm{I}$ & -25.2 & 0.73 & +39.6 & 0.38 \\
\hline $\mathrm{HDL}-\mathrm{C}<1.0 \mathrm{mmol} / \mathrm{l}$ & -13.2 & 0.65 & -53.3 & 0.26 \\
\hline Triglycerides >1.7 mmol// & +136.8 & $<0.0001$ & +90.6 & 0.009 \\
\hline Insulin $>10 \mathrm{mUl} / \mathrm{ml}$ & +28.8 & 0.36 & +30.9 & 0.35 \\
\hline Fasting plasma glucose $>5.6 \mathrm{mmol} / \mathrm{l}$ & -2.34 & 0.96 & +94.7 & 0.03 \\
\hline HOMA-IR > 2.5 & +39.2 & 0.25 & +39.8 & 0.25 \\
\hline Diabetes $^{b}$ & +28.7 & 0.69 & +139.7 & 0.09 \\
\hline Current smoker & -29.3 & 0.34 & +1.9 & 0.96 \\
\hline Hepatitis C & +12.1 & 0.85 & -129.3 & 0.08 \\
\hline Treated for hypertension & +38.7 & 0.41 & +67.8 & 0.17 \\
\hline Hypertension $^{\mathrm{C}}$ & +40.9 & 0.26 & +56.4 & 0.20 \\
\hline Stage C disease & +212.3 & 0.0002 & +45.6 & 0.47 \\
\hline \multicolumn{5}{|l|}{ Antiretroviral therapy } \\
\hline Lopinavir & & & +4.8 & 0.88 \\
\hline Atazanavir & & & +3.6 & 0.92 \\
\hline Darunavir & & & -15.0 & 0.76 \\
\hline Fosamprenavir & & & -19.3 & 0.71 \\
\hline Saquinavir & & & +77.5 & 0.50 \\
\hline
\end{tabular}

ART, antiretroviral therapy; HDL-C, HDL cholesterol; HOMA-IR, Homeostasis Model Assessment of Insulin Resistance; LDL-C, LDL cholesterol; PCSK9, proprotein convertase subtilisin/kexin type 9; $\mathrm{Pl} / \mathrm{r}$, protease inhibitors boosted with ritonavir.

${ }^{\mathrm{a}} \mathrm{BMI}>30 \mathrm{~kg} / \mathrm{m}^{3}$.

${ }^{\mathrm{b}}$ Treated for diabetes or two consecutive blood glucose at least $7 \mathrm{mmol} / \mathrm{l}$.

${ }^{\mathrm{C}}$ Treated for hypertension or SBP more than $140 \mathrm{mmHg}$ or DBP more than $90 \mathrm{mmHg}$.

$5.2 \mathrm{mmol} / \mathrm{l} \quad(P=0.02)$ and triglycerides more than $1.7 \mathrm{mmol} / \mathrm{l}(P=0.03)$ under ART (Supplementary Table S4, http://links.lww.com/QAD/B153). Inflammatory markers levels, demographic and clinical characteristics were tested but no significant associations were found (data not shown).

\section{Discussion}

In this observational study, we assessed the relationship between plasma PCSK9 levels and lipid variables, first in HIV+ ART-naive patients, then after treatment initiation with a protease inhibitor-containing regimen. Our initial hypothesis was that an increased plasma PCSK9 level would contribute to the dyslipidaemia that is frequently observed in patients on first-generation $\mathrm{PI} / \mathrm{r}$ in the clinical setting. However, we did not observe any effect of $\mathrm{PI} / \mathrm{r}$ treatment on circulating PCSK9 levels, which were already elevated in ART-naive patients versus HIVcontrols. High PCSK9 levels were associated with variables of advanced HIV infection in ART-naive HIV + patients, whereas the classical association between PCSK9 and LDL-C was disrupted. In contrast, when HIV replication was controlled by ART, the classical relationship between PCSK9 and LDL-C was restored. Altogether, these findings indicate that the expression of
PCSK9 is altered in HIV infection, possibly because of the chronic inflammatory state.

Accumulating data suggest a link between PCSK9 and inflammation. The first evidence came from the transcriptional regulation of PCSK9. Indeed, lipopolysaccharide induces hepatic PCSK9 mRNA expression in vivo in mice. TNF $\alpha$ also stimulates PCSK9 expression in a suppressor of cytokine signalling-3-dependent manner in vitro in a hepatoma cell line [17]. Finally, PCSK9 expression is induced by hepatocyte nuclear factor- $1 \alpha$, which is known to regulate the expression of acute phase proteins involved in inflammation [17]. PCSK9 inhibition results in increased lipopolysaccharide clearance, decreased inflammatory responses and improved survival following sepsis in mice $[18,19]$. In humans, circulating PCSK9 levels are increased in patients with sepsis or trauma in ICUs $[12,20]$, and patients carrying PCSK9 loss-of-function variants exhibit improved clinical outcomes during sepsis shock [21].

In contrast to a previous study [13], we did not find a significant association between plasma PCSK9 and either hs-IL-6 or hs-CRP level. Recently, a randomized study reported that treatment with a PCSK9 $\mathrm{mAb}$ (RG765) did not alter systemic markers of inflammation (hs-CRP, IL-6 or TNF $\alpha$ ) [22]. A well known inducer of PCSK9 expression is sterol depletion through activation of the 
transcription factor sterol regulatory element-binding protein 2 , and we might speculate that the sterol depletion found in ART-naive HIV+ patients may act as a potential activator of PCSK9 transcription [23]. In addition, as we found a correlation between PCSK9 levels and severity of HIV infection in untreated patients, the effect of the HIV virus itself on PCSK9 levels would be interesting to evaluate. Clearly, additional studies are warranted to identify and decipher the inflammatory and/or immune pathways governing PCSK9 expression during HIV infection.

In the current study, we found that plasma PCSK9 levels are increased in HIV+ versus HIV- patients. These findings are in accordance with those reported by Kohli et al. [13], who noted a significant increase in circulating levels of PCSK9 in patients coinfected with HIV and HCV. We also confirm the 'PCSK9 paradox' reported in Kohli et al.'s [13] study, as untreated HIV+ patients display concomitantly lower LDL-C and higher PCSK9 levels compared with HIV- patients.

One striking observation is that this so-called 'PCSK9 paradox' differentially affects LDL-C and triglyceride metabolism. Whereas the association between PCSK9 and LDL-C is disrupted in untreated HIV+ patients, circulating PCSK9 remains significantly correlated with triglycerides. These data reinforce the hypothesis for a specific role of PCSK9 in triglyceride metabolism. Regarding that point, some observations hint at a possible role for PCSK9 in the regulation of VLDL secretion. Some specific PCSK9 gain-of-function mutations promote the secretion of VLDL, possibly by stimulating the secretion of apolipoprotein B100, the major protein component of VLDL [24-26]. As insulin enhances hepatic PCSK9 expression $[27,28]$ and plasma PCSK9 levels positively associate with hepatic insulin resistance and VLDL secretion during pathophysiological conditions [29], it may be hypothesized that increased hepatic PCSK9 expression could be involved in the initiation of combined dyslipidaemia. As all lipid variables, except triglycerides, are decreased in ARTnaive HIV+ patients, it should be interesting to test the hypothesis that increased PCSK9 expression contributes to increased plasma triglycerides levels in this clinical situation. Interventional studies with PCSK9 inhibitors are warranted to further determine whether PCSK9 is involved in the regulation of triglyceride metabolism during HIV infection.

As previously reported in larger cohorts [30,31], plasma PCSK9 levels are also associated in our study with markers of glucose homeostasis, such as fasting plasma glucose and HOMA-IR, in virologically suppressed HIV+ patients. Recently, concordant Mendelian randomization studies highlighted a link between PCSK9 loss-of-function variants and the risk of diabetes [32-34], although the molecular mechanisms remain unknown.
Another unresolved issue is deciphering the underlying molecular mechanisms that sustain the disconnection between PCSK9 and LDL-C homeostasis during uncontrolled HIV infection. These mechanisms seem to be reversible, as the association between PCSK9 and LDL-C levels is restored after the control of HIV infection. Whether these mechanisms are also operative in the context of the pharmacological inhibition of PCSK9 by PCSK9 monoclonal antibodies is a critical question for clinical practice. The results from a clinical trial with a PCSK9 $\mathrm{mAb}$ in $\mathrm{HIV}+$ patients (i.e. evolocumab; NCT02833844) will help to resolve this issue. The recent advent of a $\mathrm{mAb}$ directed against PCSK9 that markedly lowers LDL-C levels with a proven benefit in reducing cardiovascular morbimortality has stimulated debate about its possible use in very high cardiovascular risk HIV + patients failing to achieve LDLC target despite maximum tolerated dose of statins and ezetimibe.

The strength of our study is the careful selection of patients who are not on lipid-lowering therapy, a situation that could have disrupted the association between PCSK9 and lipid variables. In addition, we selected HIV+ patients who were virological responders to ART, to exclude the possible effect on PCSK9 levels of persistent viral replication. The main limitation is the observational design of the study that allows us to draw some associations but not those of causation regarding PCSK9 biology in HIV.

\section{Conclusion}

We observed for the first time a dual relationship for plasma PCSK9 levels in HIV+ patients. In ART-naive patients, PCSK9 levels were elevated and positively associated with both HIV progression (low $\mathrm{CD} 4^{+}$cell counts, high HIV viral load and stage $\mathrm{C}$ disease) and plasma triglycerides, whereas after $\mathrm{PI} / \mathrm{r}$ initiation, PCSK9 levels remained elevated and were only associated with classical metabolic variables (LDL-C, triglycerides, glucose/insulin resistance). Furthermore, we observed that plasma PCSK9 levels did not change after $\mathrm{PI} / \mathrm{r}$ initiation and did not correlate with either hsIL-6 or hs-CRP. Finally, PCSK9 levels were higher in HIV + patients with or without protease inhibitorcontaining regimens compared with controls despite lower TC and LDL-C levels. Further investigations are needed to identify the factors that drive PCSK9 expression during HIV infection and to unravel the underlying molecular mechanisms that control its functional activity.

\section{Acknowledgements}

The ANRS-COPANA Cohort is funded by ANRS. The authors acknowledge the participating patients and the 
physicians, and thank Tatiana Feitoza, Abdellatif Essabani, Alain-Serge Keita for data monitoring, and Anne Persoz and Laurent Tran for data management. We thank Kalyane Bach-Ngohou for providing the samples for control patients. Sophie Rushton-Smith (MedLink Healthcare Communications Ltd) provided editorial support on the final version and was funded by the authors.

The work was supported by the Agence Nationale de Recherche sur le SIDA et les hépatites virales (ANRS). AMGEN Inc. acted as cofunder through an ANRS contract. This work also benefits from a funding from the Fondation Leducq (Grant 13CVD03).

\section{ClinicalTrials.gov Identifier: NCT03139630.}

\section{Conflicts of interest}

F.B. reports research grant form Boehringer-Ingelheim, speaker fees from AstraZeneca, Merck-Sharpe Dohme; advisory board member for Amgen, Sanofi, Gilead, ViiV Healthcare. J.C. reports research grants paid to the institution from ViiV Healthcare, Janssen and MSD, speaker fees from Viiv Healthcare, Gilead, MSD, Janssen, Chugai, Novartis. A.C. reports research grant from RESICARD (research nurses); consultant and lecture fees from AstraZeneca, Bayer Pharma, Boehringer-Ingelheim, Daiichi Sankyo, GlaxoSmithKline and SanofiAventis. B.C. has received research funding and personal fees from Sanofi and Regeneron Pharmaceuticals and Pfizer; and honoraria (advisory board, speaker fees) from Amgen, AstraZeneca, BMS, Pierre Fabre, Janssen, Eli Lilly, MSD Merck \& Co., Novo Nordisk, Novartis, Regeneron, Sanofi and Takeda. For the remaining authors, none were declared.

\section{References}

1. Brown TT, Glesby MJ. Management of the metabolic effects of HIV and HIV drugs. Nat Rev Endocrinol 2011; 8:11-21.

2. Feingold KR, Krauss RM, Pang M, Doerrler W, Jensen P, Grunfeld C. The hypertriglyceridemia of acquired immunodeficiency syndrome is associated with an increased prevalence of low density lipoprotein subclass pattern B. J Clin Endocrinol Metab 1993; 76:1423-1427.

3. Grunfeld C, Feingold KR. Metabolic disturbances and wasting in the acquired immunodeficiency syndrome. $N$ Engl / Med 1992; 327:329-337.

4. Riddler SA, Smit E, Cole SR, Li R, Chmiel JS, Dobs A, et al. Impact of HIV infection and HAART on serum lipids in men. JAMA 2003; 289:2978-2982.

5. Abifadel $M$, Varret $M$, Rabes JP, Allard D, Ouguerram K, Devillers $M$, et al. Mutations in PCSK9 cause autosomal dominant hypercholesterolemia. Nat Genet 2003; 34:154-156.

6. Cohen JC, Boerwinkle E, Mosley TH Jr, Hobbs HH. Sequence variations in PCSK9, low LDL, and protection against coronary heart disease. N Engl J Med 2006; 354:1264-1272.

7. Seidah NG, Awan Z, Chretien M, Mbikay M. PCSK9: a key modulator of cardiovascular health. Circ Res 2014; 114:1022-1036.

8. Cariou B, Si-Tayeb K, Le May C. Role of PCSK9 beyond liver involvement. Curr Opin Lipidol 2015; 26:155-161.
9. Farnier M. Proprotein convertase subtilisin kexin type $\mathbf{9}$ inhibitors: update from clinical trials to real-world experience. Curr Opin Lipidol 2016; 27:597-604.

10. Sabatine MS, Giugliano RP, Keech AC, Honarpour N, Wiviott $\mathrm{SD}$, Murphy SA, et al. Evolocumab and clinical outcomes in patients with cardiovascular disease. N Engl / Med 2017; 376:1713-1722.

11. Feingold KR, Moser AH, Shigenaga JK, Patzek SM, Grunfeld C. Inflammation stimulates the expression of PCSK9. Biochem Biophys Res Commun 2008; 374:341-344.

12. Boyd JH, Fjell CD, Russell JA, Sirounis D, Cirstea MS Walley KR. Increased plasma PCSK9 levels are associated with reduced endotoxin clearance and the development of acute organ failures during sepsis. I Innate Immun 2016; 8: 211-220.

13. Kohli P, Ganz P, Ma Y, Scherzer R, Hur S, Weigel B, et al. HIV and hepatitis C-coinfected patients have lower low-density lipoprotein cholesterol despite higher proprotein convertase subtilisin kexin 9 (PCSK9): an apparent 'PCSK9-Lipid Paradox'. J Am Heart Assoc 2016; 5:e002683.

14. Costet P, Hoffmann MM, Cariou B, Guyomarc'h Delasalle B, Konrad T, Winkler K. Plasma PCSK9 is increased by fenofibrate and atorvastatin in a nonadditive fashion in diabetic patients. Atherosclerosis 2010; 212:246-251.

15. Dray-Spira R, Legeai C, Le Den M, Boue F, Lascoux-Combe C, Simon A, et al. Burden of HIV disease and comorbidities on the chances of maintaining employment in the era of sustained combined antiretoviral therapies use. AIDS 2012; 26:207-215.

16. Matthews DR, Hosker JP, Rudenski AS, Naylor BA, Treacher DF, Turner RC. Homeostasis model assessment: insulin resistance and beta-cell function from fasting plasma glucose and insulin concentrations in man. Diabetologia 1985; 28: 412-419.

17. Ruscica M, Ricci C, Macchi C, Magni P, Cristofani R, Liu J, et al. Suppressor of cytokine signaling-3 (SOCS-3) induces proprotein convertase subtilisin kexin type 9 (PCSK9) expression in hepatic HepG2 cell line. / Biol Chem 2016; 291: 3508-3519.

18. Topchiy E, Cirstea M, Kong HJ, Boyd JH, Wang Y, Russell JA, et al. Lipopolysaccharide is cleared from the circulation by hepatocytes via the low density lipoprotein receptor. PLOS One 2016; 11:e0155030.

19. Dwivedi DJ, Grin PM, Khan M, Prat A, Zhou J, Fox-Robichaud $\mathrm{AE}$, et al. Differential expression of PCSK9 modulates infection, inflammation, and coagulation in a murine model of sepsis. Shock 2016; 46:672-680.

20. Le Bras M, Roquilly A, Deckert V, Langhi C, Feuillet F, Sebille V, et al. Plasma PCSK9 is a late biomarker of severity in patients with severe trauma injury. / Clin Endocrinol Metab 2013; 98:E732-E736.

21. Walley KR, Thain KR, Russell JA, Reilly MP, Meyer NJ, Ferguson $\mathrm{JF}$, et al. PCSK9 is a critical regulator of the innate immune response and septic shock outcome. Sci Transl Med 2014; 6:258ra143.

22. Baruch A, Mosesova S, Davis JD, Budha N, Vilimovskij A, Kahn $\mathrm{R}$, et al. Effects of RG7652, a monoclonal antibody against PCSK9, on LDL-C, LDL-C subfractions, and inflammatory biomarkers in patients at high risk of or with established coronary heart disease (from the Phase 2 EQUATOR Study). Am / Cardiol 2017; 119:1576-1583.

23. Jeong HJ, Lee HS, Kim KS, Kim YK, Yoon D, Park SW. Steroldependent regulation of proprotein convertase subtilisin/kexin type 9 expression by sterol-regulatory element binding protein2. J Lipid Res 2008; 49:399-409.

24. Sun XM, Eden ER, Tosi I, Neuwirth CK, Wile D, Naoumova RP et al. Evidence for effect of mutant PCSK9 on apolipoprotein B secretion as the cause of unusually severe dominant hypercholesterolaemia. Hum Mol Genet 2005; 14:1161-1169.

25. Sun H, Samarghandi A, Zhang N, Yao Z, Xiong M, Teng BB. Proprotein convertase subtilisin/kexin type 9 interacts with apolipoprotein B and prevents its intracellular degradation, irrespective of the low-density lipoprotein receptor. Arterioscler Thromb Vasc Biol 2012; 32:1585-1595.

26. Ouguerram K, Chetiveaux M, Zair Y, Costet P, Abifadel M, Varret $\mathrm{M}$, et al. Apolipoprotein B100 metabolism in autosomaldominant hypercholesterolemia related to mutations in PCSK9. Arterioscler Thromb Vasc Biol 2004; 24:1448-1453. 
27. Costet $\mathrm{P}$, Cariou B, Lambert G, Lalanne F, Lardeux B, Jarnoux $A L$, et al. Hepatic PCSK9 expression is regulated by nutritional status via insulin and sterol regulatory element-binding protein 1c. J Biol Chem 2006; 281:6211-6218.

28. Miao J, Manthena PV, Haas ME, Ling AV, Shin DJ, Graham MJ, et al. Role of insulin in the regulation of proprotein convertase subtilisin/kexin type 9. Arterioscler Thromb Vasc Biol 2015; 35:1589-1596.

29. Cariou B, Langhi C, Le Bras M, Bortolotti M, Le KA, Theytaz $\mathrm{F}$, et al. Plasma PCSK9 concentrations during an oral fat load and after short term high-fat, high-fat high-protein and high-fructose diets. Nutr Metab 2013; 10:4.

30. Lakoski SG, Lagace TA, Cohen JC, Horton JD, Hobbs HH. Genetic and metabolic determinants of plasma PCSK9 levels. J Clin Endocrinol Metab 2009; 94:2537-2543.
31. Baass A, Dubuc G, Tremblay M, Delvin EE, O'Loughlin J, Levy E, et al. Plasma PCSK9 is associated with age, sex, and multiple metabolic markers in a population-based sample of children and adolescents. Clin Chem 2009; 55:1637-1645.

32. Schmidt AF, Swerdlow DI, Holmes MV, Patel RS, FairhurstHunter Z, Lyall DM, et al. PCSK9 genetic variants and risk of type 2 diabetes: a Mendelian randomisation study. Lancet Diabetes Endocrinol 2017; 5:97-105.

33. Ference BA, Robinson JG, Brook RD, Catapano AL, Chapman M), Neff DR, et al. Variation in PCSK9 and HMGCR and risk of cardiovascular disease and diabetes. N Engl / Med 2016; 375:2144-2153.

34. Lotta LA, Sharp SJ, Burgess S, Perry JR, Stewart ID, Willems SM, et al. Association between low-density lipoprotein cholesterollowering genetic variants and risk of type 2 diabetes: a metaanalysis. JAMA 2016; 316:1383-1391. 